\title{
An investigation into the efficiency of disposable face masks
}

\author{
KB ROGERS \\ From the Clinical Research Centre, Watford Road, Harrow HAI 3UJ, UK
}

SUMmARY Disposable face masks used in hospitals have been assessed for the protection afforded the patient and the wearer by challenges of simulated natural conditions of stress. Operating theatre masks made of synthetic materials allow the wearer to breathe through the masks, and these have been shown to protect the patient well but the wearer slightly less. Cheaper paper masks are worn for ward duties, and of these only the Promask protected the area in front of the wearer: air does not pass through this mask, expired air is prevented from passing forward, and the wearer breathes unfiltered air. All the other paper masks tested allowed many bacteria-laden particles to pass through them.

Masks are worn for fewer ward procedures than 20 years ago, but most nursing authorities still recommend their use on the wards during the performance of many aseptic techniques even though this may be unnecessary. ${ }^{1}$ If they are used they must provide the safeguard for which they are worn, which is to prevent the nasopharyngeal organisms of the wearer infecting a patient. In a study spread over three years, nasopharyngeal carrier rates of Staphylococcus aureus varied between $29 \%$ and $42 \%$ among personnel in an operating suite, ${ }^{2}$ and it has been noted how many times people touch their noses and thus contaminate their hands. ${ }^{3}$

Masks worn by hospital staff to restrict the flow of potential pathogens from their mouths or noses are of two types, both of which are disposable. Those used during minor procedures, such as wound dressings, are usually made of paper and are not suitable for the prolonged wear necessary in an operating theatre, where more expensive masks made of one of various synthetic materials are worn; these should fit snugly over the face and cover the mouth and nose so that the wearer breathes filtered air in and out freely.

As a result of previous testing of masks at the Birmingham Children's Hospital in 1960, Smith, Nephew, and Southall evolved a disposable paper mask, the Promask, ${ }^{4}$ which was as efficient as the linen mask of that time which had a cellophane or paper insert. One of the two layers of paper in the original Promask was similar to thin airmail paper

Received for publication 18 March 1980 through which air could not pass; this layer has now been replaced by one of tissulate, made of laminated polyethylene and polypropylene.

Few, if any, organisms would be expelled from their mouths if staff did not talk, or talked very quietly, while performing minor procedures such as wound dressings. Because it is impossible to guarantee that explosive sounds will not be made at such times, the efficiency of disposable masks was examined by challenges of simulated natural conditions of stress to which they might be subjected.

\section{Methods}

The masks were worn on the face or tied over the top of the tube, with an internal diameter of $2.8 \mathrm{~cm}$, leading to the slit of the Casella air sampling machine. ${ }^{5}$ The makers of a mask that proved inefficient asked for it to be tested on a head to allow a larger area of the mask to act as a filter. Madame Tussauds Ltd loaned life-sized heads, but unfiltered air was sucked through gaps that appeared where the masks' edges could not sink into the hard wax cheeks. These heads were not used when it was found that a good and more consistent filtering area was provided by tying the masks across the top of a glass funnel. In the tests that are now reported, the masks have been examined when worn on the face and fixed over a funnel.

A $7.8 \mathrm{~cm}$ diameter glass funnel was cut so that its narrowest portion measured $2.3 \mathrm{~cm}$, and this narrow end was cemented into a $670 \mathrm{~cm}$ long plastic tube with an internal diameter of $3 \mathrm{~cm}$. The plastic tubing 
was pushed over the metal tube leading to the slit of the air sampler and fixed to it with a clip. A single, loudly spoken 'Tut' $(T)$ or a single explosive 'spraying spit' ${ }^{6}$ or 'raspberry' $(R)$ was directed at the mouth of the funnel from a distance of about $200 \mathrm{~mm}$. After testing a mask when it was fixed over the funnel, the mask was examined to ensure that it had not been torn: if it had been damaged the tests were repeated using a new mask. Nutrient blood agar plates were rotated in the sampler for half a minute and incubated at $37^{\circ}$ for 18 hours, when the colonies were counted.

\section{Results}

The first experiments were made in a room $700 \mathrm{~cm}$ long, $244 \mathrm{~cm}$ wide, and $290 \mathrm{~cm}$ high, approximately $50 \mathrm{~m}^{3}$ in size, the windows of which were kept shut. On the same day several masks, including the Promask and good theatre masks, were tested when worn on the face, and none appeared as efficient as when tested in Birmingham.

The air of the room was sampled before any tests were made on the masks; six plates, each exposed for half a minute, gave an average count of 4.8 colonies. When the air was sampled between $T$ and $R$ tests the average count on 40 plates was 53.7 . It was, therefore, impossible to judge the efficiency of a mask tested on the face in this room as the suspended bacteria-laden particles from previous tests were being deposited on the plates.

When the air in this room was sucked through the masks after they had been fixed on the funnel, many of these suspended, and therefore presumably small, bacteria-laden particles passed through the paper masks, but few through three of the good theatre masks (Table 1).

*Liberty Products Inc.
The experiments were repeated in a Bio-Hood Mark IV M Safety Cabinet,* which Clark $^{7}$ had proved was very efficient. The slit sampler was placed in the cabinet, and the funnel on the end of the plastic tube was pulled down so that it was always at the same marked position just outside the cabinet's safety screen. After the T or R challenge had been made, the funnel was replaced in the safety cabinet. The air of this cabinet never grew organisms, even when sampled after a control $\mathbf{R}$ test, which gave a very high count. This series of experiments confirmed the earlier work showing that the Promask did not allow bacteria-laden particles to pass forward and that the modern masks used in theatres were uniformly efficient. It also confirmed the relative inefficiency of other paper and Aseptex masks.

The results of the investigations performed in the cabinet are summarised in the Figure where the ordinate gives the number of colonies grown. Each column represents one test on a mask, or a control test. The results from tests when the masks were worn on the face show the protection offered to a patient, and those when the masks were on the funnel show the protection given to the wearer. Pairs of $T$ and $R$ control tests were made, when no mask was worn or put over the funnel, on each day that masks were tested. Table 2 shows the percentage of the control count that was able to pass through the masks. The most stringent tests appear to have been those made on the funnel and the $R$ tests.

These histograms demonstrate that most of the tests through masks produced lower counts than the controls, probably because large particles are trapped by the masks which provide a coarse baffle, even though the bad masks fail to act as good filters.

When the tapes at the bottom of a mask were $34 \mathrm{~cm}$ or less in length it was difficult to tie the mask on the head, but if they were at least $38 \mathrm{~cm}$ long it was easy to do so.

Table 1 Masks fixed over funnel in room: number of colonies counted in half a minute

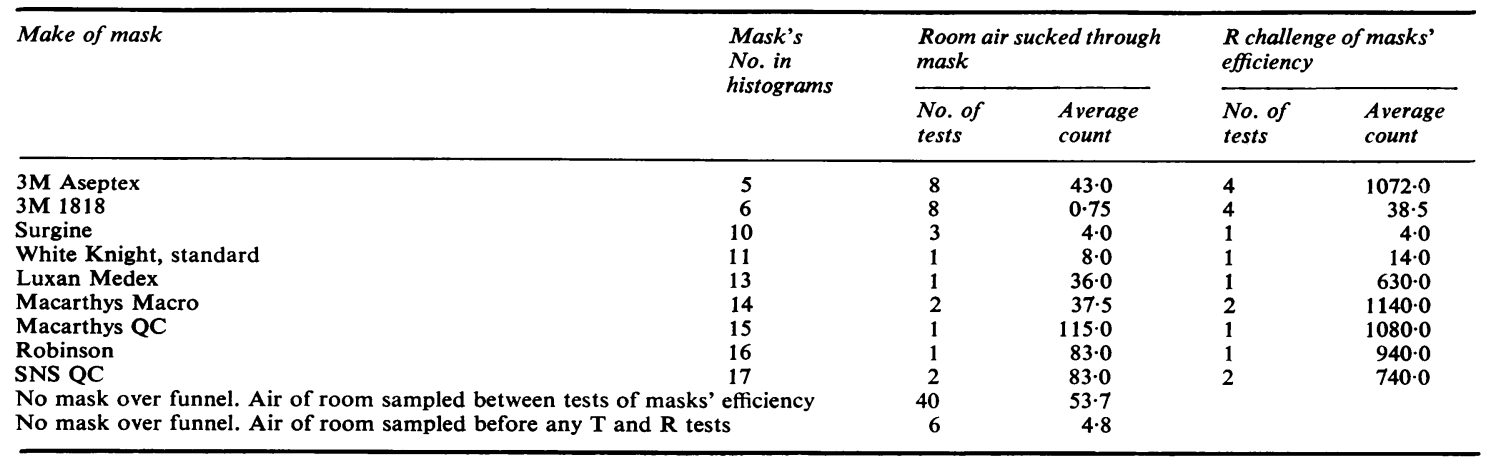




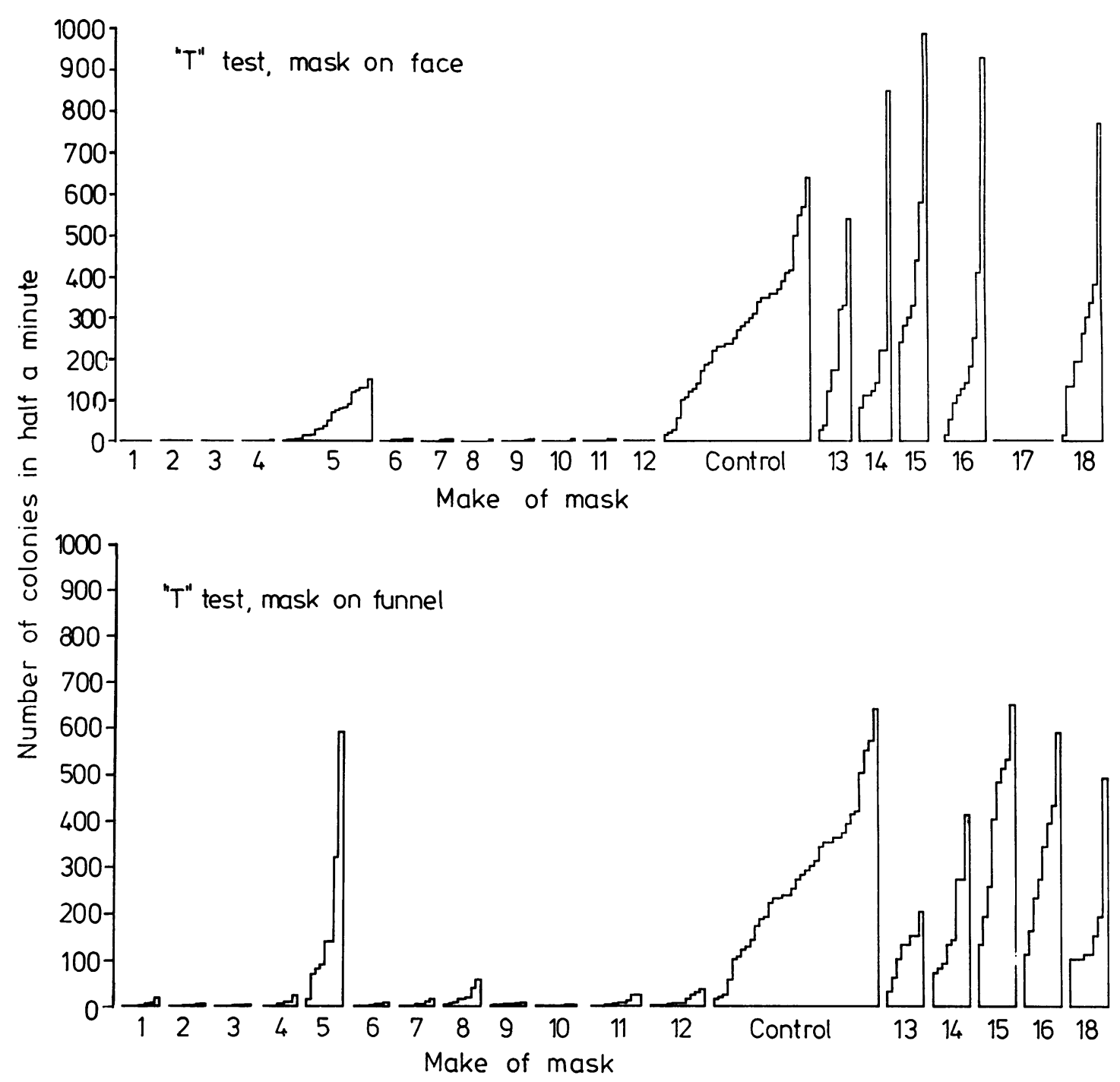

Key to masks in histograms

$\begin{array}{cl}\text { Number } & \text { Make of mask } \\ 1 & \text { Bard international } \\ 2 & \text { Bard Vigilon } \\ 3 & \text { Deseret E-Z Breathe } \\ 4 & \text { Deseret no glass } \\ 5 & \text { 3M Aseptex } \\ 6 & \text { 3M 1818 } \\ 7 & \text { 3M Safety } \\ 8 & \text { Seward Medical } \\ 9 & \text { Seward no glass } \\ 10 & \text { Surgine }\end{array}$

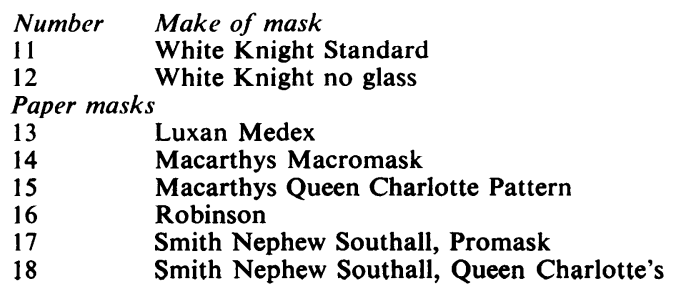




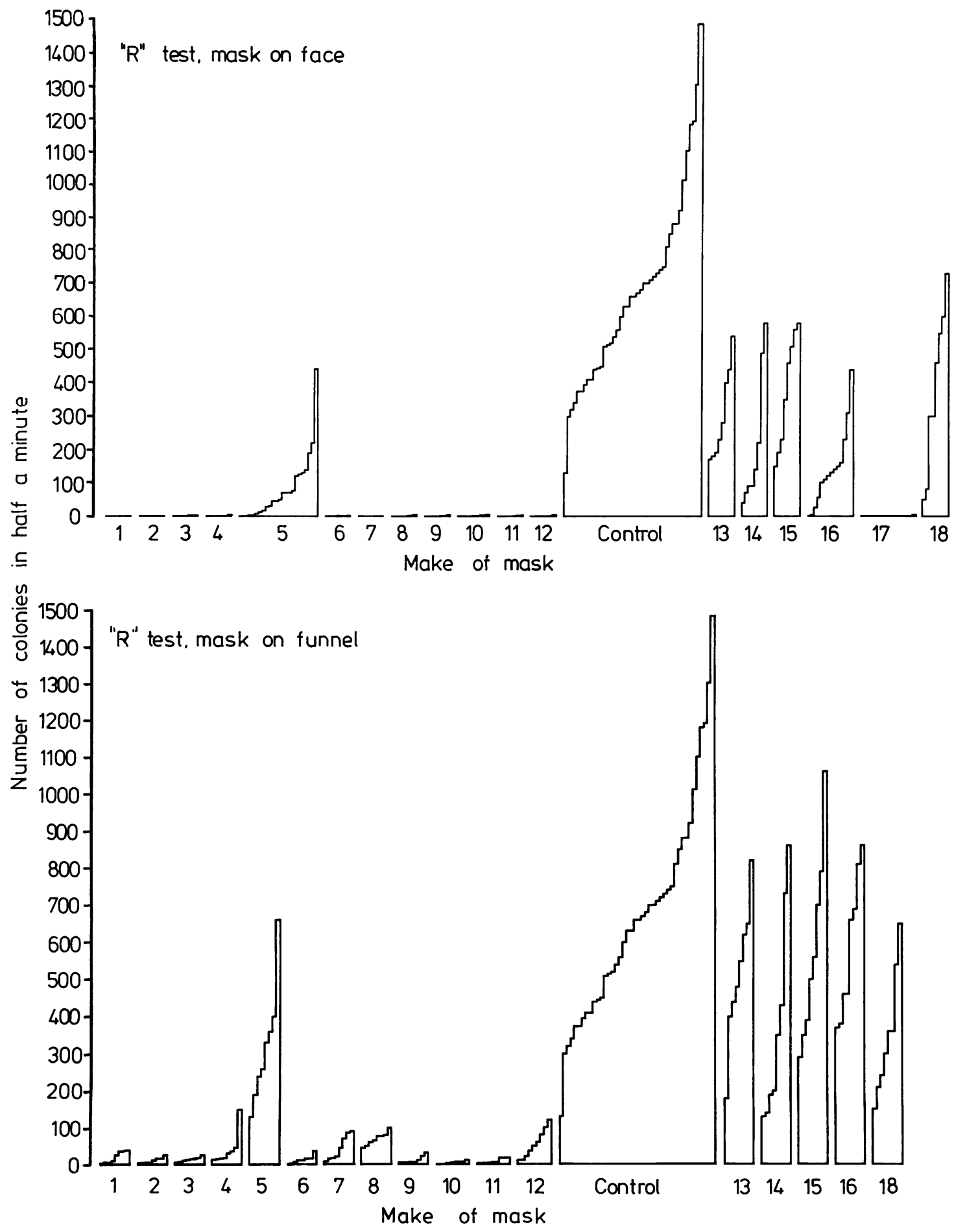


Table 2 Percentage of control counts passing through masks, demonstrating efficiency of each mask

\begin{tabular}{|c|c|c|c|c|c|}
\hline \multirow{3}{*}{$\begin{array}{l}\text { No. } \\
\text { in } \\
\text { histograms }\end{array}$} & \multirow[t]{3}{*}{ Make of mask } & \multicolumn{4}{|c|}{ Position of mask } \\
\hline & & \multicolumn{2}{|c|}{$\begin{array}{l}\text { On face } \\
\text { (type of challenge) }\end{array}$} & \multicolumn{2}{|c|}{$\begin{array}{l}\text { On funnel } \\
\text { (type of challenge) }\end{array}$} \\
\hline & & $T$ & $\boldsymbol{R}$ & $T$ & $R$ \\
\hline 1 & Bard International & 0 & 0.02 & 1.9 & $3 \cdot 1$ \\
\hline 2 & Bard Vigilon & 0.05 & 0 & 0.7 & 1.6 \\
\hline 3 & Deseret E-Z Breathe & 0 & 0.06 & 0.6 & $2 \cdot 0$ \\
\hline 4 & Deseret no glass & 0.05 & 0.02 & $2 \cdot 6$ & 6.0 \\
\hline 5 & 3M Aseptex & $21 \cdot 1$ & $11 \cdot 6$ & $77 \cdot 2$ & $47 \cdot 3$ \\
\hline 6 & $3 \mathrm{M} 1818$ & 0.1 & 0.07 & 0.9 & 1.8 \\
\hline 7 & 3M Safety & $0 \cdot 2$ & 0.04 & $7 \cdot 7$ & 6.5 \\
\hline 8 & Seward Medical & 0.05 & 0 & $7 \cdot 7$ & $10 \cdot 1$ \\
\hline 9 & Seward no glass & $1 \cdot 1$ & 0.07 & 1.4 & $1 \cdot 7$ \\
\hline 10 & Surgine & $0 \cdot 1$ & $1 \cdot 2$ & 0.3 & 0.6 \\
\hline 11 & White Knight Standard & 0.4 & 0.04 & $2 \cdot 6$ & $1 \cdot 3$ \\
\hline 12 & White Knight no glass & 0 & 0.02 & $4 \cdot 1$ & $10 \cdot 7$ \\
\hline \multicolumn{6}{|c|}{ 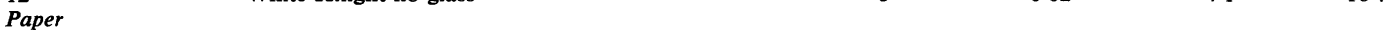 } \\
\hline 13 & Luxan Medex & $78 \cdot 6$ & $44 \cdot 7$ & $44 \cdot 2$ & $72 \cdot 2$ \\
\hline 14 & Macarthys Macromask & 86.0 & $31 \cdot 7$ & $67 \cdot 9$ & $55 \cdot 7$ \\
\hline 15 & Macarthys, Queen Charlotte & $202 \cdot 8$ & $55 \cdot 8$ & $146 \cdot 7$ & $85 \cdot 6$ \\
\hline 16 & Robinson & $71 \cdot 2$ & 20.7 & $117 \cdot 2$ & $86 \cdot 3$ \\
\hline 17 & Smith Nephew Southall, Promask & 0 & 0 & \multicolumn{2}{|c|}{ (Tests not possible) } \\
\hline 18 & Smith Nephew Southall, Queen Charlotte's & 100 & $56 \cdot 3$ & 62.8 & $51 \cdot 7$ \\
\hline
\end{tabular}

\section{Discussion}

The techniques used to test mask efficiency have varied widely, the mask being placed in many different situations where they have been challenged by a variety of natural stresses as well as by artificial loads of bacteria and a virus, and the organisms have been harvested by several techniques.

Volunteers have challenged masks, when their heads were placed in specially constructed cabinets, ${ }^{8-12}$ by quiet breathing and speaking ${ }^{8}$ and enunciating, ${ }^{9}$ or shouting, ${ }^{12}$ 'sing and chew'. The bacteria passing through the mask have been collected by the Andersen cascade sampler, ${ }^{13}$ the Bourdillon slit sampler, ${ }^{5}$ or on settle plates. ${ }^{814}$ Sterile gauze, impregnated with a $1 \%$ solution of sodium alginate, has been placed in a frame suspended on a modified head mirror in front of a surgeon's face while operating; after an operation the gauze was pressed on and then removed from a culture plate which was incubated. ${ }^{15}$

Masks on manikin heads mounted in a chamber have been challenged with an aerosol containing Bacillus stearothermophilus ${ }^{10}$ or in specially constructed chambers where their filtering ability was challenged by an aerosolised bacterial suspension of Serratia marcescens, ${ }^{216}$ Staphylococcus aureus, ${ }^{17}$ or a virus. ${ }^{17}$

The Madsen's ${ }^{10}$ showed that the Aseptex mask gave $98 \%$, and Quesnel ${ }^{11}$ that it provided $92.6 \%$, protection of the patient. The results presented in the Figure and in Table 2 show this mask to be relatively inefficient, a result supported by Clark's ${ }^{18}$ Schlieren cine photography, in which jets of air are seen coming through small holes in this mask. Nicholes ${ }^{19}$ found that the Aseptex mask seldom showed a filtration efficiency greater than $25 \%$.

These investigations were spread over several weeks so that many control $\mathrm{T}$ and $\mathrm{R}$ tests were made. The challenges have not been uniform, but the scatter would not seem much, if any, worse than those of other workers. Sedimentation plate counts of a masked volunteer ranged from 4 to 23 , and the Andersen sampler plates from another, whose control small particle counts were 42-347, ranged from 3 to 64 when masked. Masks challenged on manikin heads had control counts ranging from 232 to 2432, and the counts when testing an Aseptex mask were from 3 to 48 , from 2 to 34 with a Filtron, and from 3 to 104 with a Bardic Deseret mask. ${ }^{10}$

Thomas, ${ }^{14}$ who used three techniques to test masks, commented on the great scatter of his results. He noted that the slit sampler provided the most reproducible results and found it convenient to fix his masks over a funnel connected to a Bourdillon sampler.

Ford and Peterson ${ }^{16}$ stated that some wearers found that unfiltering masks were most uncomfortable. A Promask was worn without discomfort for 3 hours while performing routine work. The mask was challenged by a series of $\mathbf{R}$ tests during this time, when trips between the wards and the laboratories involved walking at least $500 \mathrm{~m}$ and going up and down at least 300 stairs. No organism came through the mask even after 3 hours, when the outside, and then the inside, of the mask were pressed on separate blood agar plates. The outside yielded three colonies of Staphylococcus epidermidis, and the inside a sheet 
of nasopharyngeal organisms.

There has been a steady move to the use of disposable equipment. ${ }^{16}$ Anyone who has seen staff disentangling the tapes and reassembling autoclaved linen masks would consider this change an advantage. But the disposable masks should be better than those used previously; they should fit comfortably, be easy to breathe through, and lack allergenicity. ${ }^{2}$ Except for the Promask, all the disposable paper masks I have tested have been inefficient.

The good theatre type masks, made of synthetic fibres or fibre glass, and the Promask will protect the patients extremely well. None would be perfect for a wearer in a very heavily contaminated atmosphere. None of the paper masks, except the Promask, nor the Aseptex mask provide good protection of the patient, and none gives good protection of the wearer; the wearer of a Promask breathes unfiltered air.

The masks tested and reported on in this communication have all been used in National Health Service hospitals. Enquiries from one maker produced replies that were the final stimulus to the production of this article. The marketing manager wrote:

'... hospital contracts having been placed with the cheapest source ...' '... as long as people continue to buy the cheaper mask, which is apparently widely accepted, my firm would continue to sell it' ... 'Masks of much inferior quality to ours are being imported and sold from Hong Kong and the fact that they are probably useless seems to have little effect upon the Central Contracting Authorities who appear to buy purely on a price basis'.

Central Contracting Authorities should not purchase articles such as disposable paper face masks on a cost basis only. Except for the Promask, the cheap paper masks are potentially dangerous as they produce a false sense of security, and under stress they prevent few bacteria-laden particles travelling from the wearer's mouth towards the patient.

I am grateful to Drs RP Clark, JM Dolby, and DAJ Tyrrell, of the Clinical Research Centre, for the generous help they have each given at different times; and the Department of Medical Illustration for assistance in reproducing the illustrations. I also thank the manufacturers for the supplies of the masks tested, and Madame Tussauds Ltd for the loan of the wax, life-size heads.
References

${ }^{1}$ Hare R. To mask or not to mask. Nurs Times 1962;59:715.

${ }^{2}$ Ford CR, Peterson DE, Mitchell CR. An appraisal of the role of surgical face masks. Am J Surg 1962;113:787-90

${ }^{3}$ Hare R. The transmission of respiratory infections. Proc $R$ Soc Med 1964;57:221-9.

${ }^{4}$ Rogers KB. A disposable face mask. Lancet 1960;1:931.

${ }^{5}$ Bourdillon RB, Lidwell OM, Thomas JC. A slit sampler for collecting and counting airborne bacteria. J Hyg (Camb) $1941 ; 41: 197-224$.

6 Studies in air hygiene. Spec Rep Ser Med Res Coun Lond No. 262, 1948;63.

${ }^{7}$ Clark RP. Personal communication 1978.

${ }^{8}$ Shooter RA, Smith MA, Hunter CJW. A study of surgical masks. Br J Surg 1959;47:246-9.

${ }^{9}$ Greene VW, Vesley D. Methods for evaluating effectiveness of surgical masks. J Bacteriol 1962;83:663-6.

${ }^{10}$ Madsen PO, Madsen RE. A study of disposable surgical masks. Am J Surg 1967;114:432-5.

${ }^{11}$ Quesnel LB. The efficiency of surgical masks of varying design and composition. Br J Surg 1975;62:936-40.

12 Sommers JR. Surgical face masks. Bacterial filtration efficiencies study. Surgicos $1978 ; 55: 481-2$.

13 Andersen AA. New sampler for the collection, sizing and enumeration of viable airborne particles. $J$ Bacteriol 1958;76:471-84.

14 Thomas CGA. Efficiency of surgical masks in use in hospital wards. Guy's Hosp Rep 1961 ;110:157-67.

${ }^{15}$ Freake R, Abbott L. Method for testing the bacteriological efficiency of surgical masks in the operating theatre. Lancet 1966;1 :78-9.

${ }^{16}$ Ford CR, Peterson DE. The efficiency of surgical face masks. Am J Surg 1963;106:954-7.

${ }^{17}$ Nicholes PS. Comparative evaluation of a new surgical mask medium. Surg Gynecol Obstet 1964;118:579-83.

${ }^{18}$ Clark RP. Film of surgeons' clothing. In The Human Microenvironment and Airborne Infection 1977. Available from Dr RP Clark at Laboratory of Aerobiology, Clinical Research Centre, Harrow.

19 Nicholes PS. Personal communication 1965.

Requests for reprints to: $\mathrm{Dr}$ KB Rogers, Queen Elizabeth Hospital for Children, Hackney Road, London E2 8PS, UK.

\section{Addendum}

Since this paper was written another theatre mask and another paper mask have been tested; neither is a good mask and the percentage of control counts that passed through each mask is as follows:

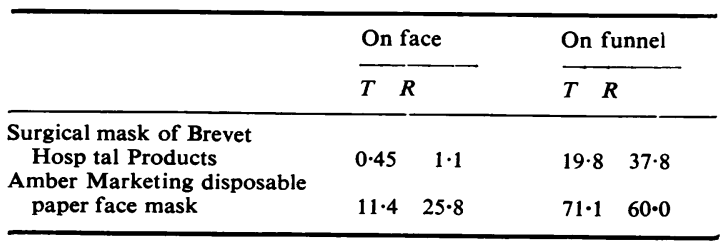

